\section{Observations of $\mathrm{A0620}-00$ at 962 and $151 \mathrm{MHz}$}

Following the discovery by the X-ray astronomy group at Leicester University of a large increase in the X-ray flux of A0620 - 00, observations at $962 \mathrm{MHz}$ and $151 \mathrm{MHz}$ have been made at Jodrell Bank. During the period August 16-29, observations were made at $962 \mathrm{MHz}$ with the MK II-MK III long baseline radio-link interferometer. This instrument has a spacing of $23.7 \mathrm{~km}$ giving a lobe size of $\sim 3^{\prime \prime}$ at this frequency. The radio-link is phase compensated so that integrations can be made for several hours.

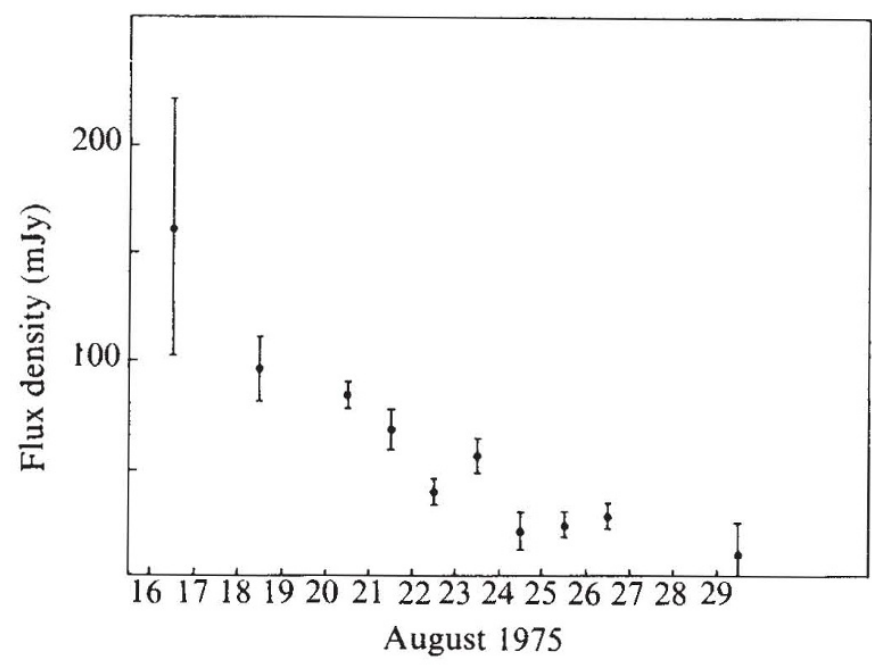

Fig. 1 Flux density of $\mathrm{A0620}-00$ at $962 \mathrm{MHz}$ with a resolution of $3^{\prime \prime}$

On August 16 and 18, the instrument was used to survey an area $0.5^{\circ}$ by $0.5^{\circ}$ centred on the position of the X-ray source ${ }^{1}$. For a source at declination $0^{\circ}$ the synthesised beam after integrating from hour angle $1.5-3.5 \mathrm{~h}$ is $5^{\prime \prime}$ in right ascension and $30^{\prime}$ in declination. In the area surveyed only one source, at the extreme edge of the field, was significantly above the noise level. On August 19 the position of an optically variable object, possibly associated with the X-ray source, was communicated to us $^{2}$. The right ascension of this object and the observed radio source agreed to within $6^{\prime \prime}$ making an association likely. Further observations were therefore restricted to a smaller area of sky surrounding the position of the radio source and optical object. Thus a wider bandwidth could be used, giving an increased sensitivity and enabling the position to be measured with greater accuracy. With the $4-\mathrm{MHz}$ bandwidth the r.m.s. noise on an amplitude measurement after a 2-h integration was $6 \mathrm{mJy}$. The position measured on August 18 was: $\alpha=06 \mathrm{~h}$ $20 \min 11.4 \pm 0.27 \mathrm{~s} ; \delta=-0^{\circ} 17 \pm 7^{\prime}$ (1950.0). Subsequently, more accurate measurements of the $\mathrm{X}$-ray source position confirmed the radio identification ${ }^{3,4}$. No significant variation of fringe amplitude with hour angle was detected during the observations, thus indicating that the angular size of the radio source was less than $3^{\prime \prime}$. Measurements of the flux density of the radio source were continued until August 29 and showed a decreasing intensity with time as shown in Fig. 1.

During August 12-15 observations were also made at 962 $\mathrm{MHz}$ using the MK II radio telescope. Total power measurements indicated that the flux density of the source was less than $5 \mathrm{Jy}$. On August 17, 20, 23 and 24 observations were made using the MK IA-Defford radio-link interferometer at $151 \mathrm{MHz}$. This instrument has a baseline of $127 \mathrm{~km}$ which gives a lobe size of $3^{\prime \prime}$ at this frequency. No radio emission was observed down to a sensitivity limit of $250 \mathrm{mJy}$. At this frequency, however, interstellar scattering may increase the apparent angular size of a source near the galactic plane, as in the case of Cyg $\mathrm{X}-3$ (ref. 5), and this could explain why no source was observed in the present observations at $151 \mathrm{MHz}$.

If the decrease of flux density shown in Fig. 1 is expressed as an exponential decay, the time for the flux density to fall by a factor of $1 / e$ is $5.2 \mathrm{~d}$. The data may also be fitted to a power law of the form $\mathrm{t}^{-3 \pm 1}$ on the assumption that the outburst originated on August 3.0 UT. On the simple van der Laan model $^{6}$ of uniform expansion of an optically thin cloud of relativistic electrons, this would correspond to a radio spectral index, $\alpha$, of $-0.25 \pm 0.25$ where $\alpha$ is defined by $S=S_{0} v^{+\alpha}$.
R. J. Davis
M. R. EDWARDS
I. MORISON
R. E. SPENCER

University of Manchester,

Nuffield Radıo Astronomy Laboratories,

Jodrell Bank, Cheshire, UK

Received September 8; accepted September 22, 1975.

${ }^{1}$ Elvis, M., Page, C. G., Pounds, K. A., Ricketts, M. J., and Turner, M. J., Nature 257, 656-657 (1975).

Boley, F., and Wolfson, R., IAU Circ. No. 2819.

3 Matilsky, T., IAU Circ., No. 2819.

4 Eyles, C. J., Skinner, G. K., and Willmore, A. P., IAU Circ., No. 2822.
5 Anderson, B., et al., Nature phys. Sci., 239, 117 (1972).

6 van der Laan, H., Nature, 211, 1 i 31 (1966).

The optical counterpart of $\mathrm{A0620}-00$ before its eruption

AN accurate position of the optical counterpart of A0620-00 discovered by Boley and Wolfson ${ }^{1}$ has made possible the identification of the image of the star on the Palomar Observatory Sky Survey (POSS) charts.

The position was measured on two 10-min exposures on Eastman Kodak IIa0 plates taken on the 13-inch astrographic telescope at Herstmonceux on August 27-28 and 28-29, 1975 relative to standard positions of AGK3 stars, using a Zeiss $x-y$ measuring engine. It was reduced using the methods described by Murray, Tucker and Clements ${ }^{2}$. The mean position of the Boley-Wolfson star which appears at $B \sim 12.2$ on both plates is: $\alpha=06 \mathrm{~h} 20 \mathrm{~min} 11.176 \pm 0.021 \mathrm{~s} ; \delta=-00^{\circ}$ $19^{\prime} 10.80 \pm 0.32^{\prime \prime}(1950.0)$, where r.m.s. errors are quoted. There is a substantial contribution to these errors from the uncertainty of the system defined by the AGK3 stars and from the possible effects of refraction if the identification has a different colour from the AGK3 stars. The right ascension lies about 3 s.d. $\left(1.20 \pm 0.35^{\prime \prime}\right)$ from that of the radio object measured at the Mullard Radio Astronomy Observatory ${ }^{3}$. The position is, however, within the X-ray error boxes measured by the Leicester ${ }^{4}$ and Birmingham-Mullard Space Science Laboratory ${ }^{5}$ experiments on Ariel $\mathrm{V}$ and by the Massachusetts Institute of Technology experiment ${ }^{6}$ on SAS-3.

The positions of a number of stars on the POSS charts (from originals taken on November 18-19 1955) were also determined with respect to the same AGK3 standards from measurements made with a Faul Coradi Coradigraph $x-y$ machine. Similar reductions showed that one star, identified in Fig. 1, lies at: $\alpha=06$ h $20 \min 11.202 \pm 0.023 \mathrm{~s} ; \delta=-00^{\circ} 19^{\prime} 10.45+0.35^{\prime \prime}$ (1950.0).

In comparing these two positions the errors of the positional system defined by the AGK3 stars cancel and so the difference in position has smaller errors than the appropriate combination of the errors in the positions. The differences between the measurements obtained on the POSS and the 13-inch plates are: $\Delta \alpha=+0.39 \pm 0.38^{\prime \prime}$ and $\Delta \delta=+0.35 \pm 0.38^{\prime \prime}$. Since the positions agree very well and the next nearest object on the POSS is $\sim 15^{\prime \prime}$ away, the star identified in Fig. 1 is very likely to be the optical counterpart before its eruption.

Eye estimates on the POSS give magnitudes in 1955 for the counterpart of $B \sim 20.5, B-R \sim 3.6$ where the errors are likely 